\title{
Management of schizophrenia in conditions of high security
}

\author{
Lindsay D. G. Thomson
}

\section{High-security psychiatric care in the UK}

There are four high-security hospitals in the UK: Broadmoor Hospital in Berkshire (founded in 1863), Rampton Hospital in Nottinghamshire (1914), Ashworth Hospital in Merseyside (1990), which opened following the amalgamation of Park Lane (1974) and Mosside Hospitals (1913), and the State Hospital at Carstairs in Lanarkshire (1948). The first three are known as the special hospitals and serve England and Wales. The latter offers a special security service, combining high and medium secure care, for the whole of Scotland and Northern Ireland - there is no form of medium secure psychiatric provision in these countries. All four hospitals provide care for patients with mental disorders and dangerous, violent or criminal propensities. There are approximately 1550 beds in these facilities and all patients are formally detained under mental health or criminal legislation. The special hospitals are currently administered by the high-security psychiatric services commissioning team, managed locally as individual authorities, although in the future it is intended to integrate them more fully into the National Health Service (NHS) and to organise both high and medium secure services at a regional level. Carstairs is administered by the State Hospital Board for Scotland which has the status of a special health board.

The population of the high-security hospitals has been extensively described (Maden et al, 1993; Thomson et al, 1997; Taylor et al, 1998). Both north and south of the border $30-50 \%$ of their patients have been said not to require high-security psychiatric care. There is no strict definition of what is meant by high security but all of these hospitals have a secure perimeter fence with powerful lighting, cameras and motion detectors. Security procedures vary in each institution but most have airport-style security measures such as X-ray screening and metal detectors. Personal alarms, air-lock entries to wards and security policies, for example on random searches, are routine. Good staff/patient relationships and staff training are crucial to ensure safety and accurate risk assessment. Mandatory directions regarding safety and security in Ashworth, Broadmoor and Rampton Hospitals were issued by the Department of Health last year (National Health Service Executive, 1999). These cover subjects such as searching of patients and staff, and access to telephones, post and computers.

\section{Schizophrenia in high-security hospitals}

Schizophrenia is the core condition in the highsecurity hospitals. Studies found that 169 patients $(70.1 \%)$ in the State Hospital and $818(47 \%)$ in the special hospitals had schizophrenia. Patients with personality disorder account for the difference between the two populations. In the State Hospital only 13 patients $(5.4 \%)$ had a primary diagnosis of personality disorder compared with 713 (41\%) in the special hospitals. This reflects a difference in psychiatric practice. Comorbidity in both groups was common (see Table 1).

Lindsay Thomson is Senior Lecturer in Forensic Psychiatry at the University of Edinburgh (Department of Psychiatry, Kennedy Tower, Morningside Park, Edinburgh EH10 5HF) and Honorary Consultant Psychiatrist at the State Hospital, Carstairs. Her research interests include needs assessment in forensic settings, evaluation of risk, and substance misuse in forensic populations. 
Table 1. Patients with schizophrenia and comorbidity

$\left.\begin{array}{lcc}\text { Diagnoses } & \text { Special hospitals } & \text { State Hospital } \\ & n(\%) & (\%)\end{array}\right)$

1. Acquired brain damage $(n=3)$; learning disability and substance misuse $(n=1)$; learning disability and sexual deviation $(n=1)$; personality disorder and learning disability $(n=6)$; personality disorder and sexual deviation $(n=4)$; personality disorder with affective neurosis $(n=1)$; with substance misuse $(n=2)$.

2. Schizophrenia and organic brain syndrome $(n=2)$; schizophrenia and personality disorder and organic brain syndrome $(n=2)$; schizophrenia and personality disorder and learning disability $(n=3)$; schizophrenia and organic brain damage and substance misuse $(n=2)$; schizophrenia and learning disability and substance misuse $(n=1)$.

The two high-security populations are broadly similar (see Table 2).

A comparison of patients with schizophrenia in high security with those in the community found poorer educational attainment, a greater personal and family history of substance misuse, and a much more extensive forensic history in the high-security patients. In addition, they were more likely to have had developmental problems in childhood and to suffer from a chronic illness. Significantly more were male (Miller et al, 2000).

\section{Schizophrenia, crime and violence}

As patients are admitted to a high-security setting because of an offence or aggression, it is relevant to review the evidence for associations between mental disorders, crime and violence. Swanson et al (1990) examined violence in psychiatric disorders in the community using evidence from the Epidemiologic Catchment Area survey. Responders $(n=368)$ reported that they had been violent during the past 12 months and $55.5 \%$ of these had a psychiatric disorder, mostly substance misuse. The prevalences of affective disorders $(9.37 \%$ v. $2.95 \%)$ and of schizophrenia $(3.92 \%$ v. $1.03 \%)$ were three times greater in violent than non-violent responders. The model for predictors of violence from this study included gender, age, socio-economic status, substance misuse and major mental illness.

Birth cohort studies have also found an association between schizophrenia and criminal acts. The
Northern Finnish Birth Cohort Study ( $n=12$ 058), which controlled for socio-economic status, found that individuals with schizophrenia were three times more likely to be convicted of a crime and seven times more likely to be convicted of a violent crime (Tiihonen et al, 1997). The Danish Birth Cohort Study $(n=324401)$ followed subjects to the age of 43 and found an association between admission to psychiatric hospital and criminal conviction (Hodgins et al, 1996). Other researchers have chosen to examine schizophrenia specifically in association with

$\begin{array}{lcc}\begin{array}{l}\text { Table 2. Description of } \\ \text { population }\end{array} & \begin{array}{c}\text { Special } \\ \text { hospitals }\end{array} & \begin{array}{c}\text { State } \\ \text { Hospital }\end{array} \\ & 32.0(9.1) & 30.8(8.6) \\ \text { Age (s.d.) } & 89 & 89 \\ \text { Male (\%) } & 70 & 87 \\ \text { Single (\%) } & 84 & 77 \\ \text { Unemployed (\%) } & & \\ \text { Admission source } & 40 & 36 \\ \text { Other psychiatric facility (\%) } & 36 & 44 \\ \text { Court (\%) } & 23 & 20 \\ \text { Prison (\%) } & 2 & - \\ \text { Other (\%) } & & \\ \text { Index offence } & 29 & 23 \\ \text { Homicide (\%) } & 33 & 17 \\ \text { Assault (\%) } & 6 & 5 \\ \text { Sexual assault (\%) } & 6 & 2 \\ \text { Arson (\%) } & 11 & 13 \\ \text { Others (\%) } & 15 & 40 \\ \text { None (\%) } & 54 & 83 \\ \text { Previous convictions (\%) } & 54 \\ \text { Mean time in high security, } & & \\ \text { years (median) } & 6.89(5.27) & 5.27(2.97) \\ & & \end{array}$


criminal or dangerous behaviour. Humphreys et al (1992) examined dangerous behaviour preceding first admission for schizophrenia and specifically excluded patients with a history of drug or alcohol misuse. One-fifth of cases were found to have behaved in a life-threatening manner towards others prior to their initial admission. Wessely et al (1994) looked at the criminological histories of incident cases of schizophrenia and found that violence in male patients with schizophrenia was 3.8 times greater than in other mentally disordered groups. In women this three-fold increase applied to all forms of crime. They found that the strongest predictors of crime were gender, ethnicity, unemployment, younger age at onset, substance misuse and a history of previous offending. A Swiss study (Modestin \& Ammann, 1996) examined male in-patients with schizophrenia $(n=1265)$ and found that $36 \%$ had a criminal history compared with $15 \%$ of the general male population. However, when they controlled for marital status, job and size of community of residence, they found that the control population had a criminal history in $34 \%$ of cases. Important differences were found between the schizophrenia and control groups when the type of conviction was analysed, in that the schizophrenia group had five times more convictions for violence, three times more for drug offences and 2.5 times more for property offences. The control group had an excess of traffic offences.

Instead of looking at the specific diagnosis and its relationship to crime, some researchers have examined specific crimes and looked for associations with mental disorder. Eronen et al (1996) investigated mental disorders and homicidal behaviour in Finland $(n=693)$ where $95 \%$ of cases are solved. They found that the odds ratio of a homicide being committed by a person with schizophrenia was 8fold for men and 6.5-fold for women.

Population studies, patient studies, and studies of specific crimes or diagnosis all, therefore, show an association between schizophrenia and crime, in particular violence. In the USA, however, the McArthur Violence Risk Assessment Study looked at people between the ages of 18 and 40 discharged from acute psychiatric in-patient facilities $(n=1136)$ and compared their levels of violence to others living in the same neighbourhood (Steadman et al, 1998). Data on violence were gathered by self-report, from third-party informants and from police and hospital records every 10 weeks for a period of a year for the patient group and for one 10-week period for the control group. No difference was found in levels of violence between the schizophrenia cohort and the neighbourhood controls. Link \& Stueve (1998) have suggested that this finding is not inconsistent with previous studies because of the importance of timing. They argue that it is in the acute stage of a psychotic episode that violence is more likely and that by the nature of this research these patients were excluded. Others have argued that the in-patient facilities from which subjects were recruited are not typical of the institutions in which many patients with schizophrenia find themselves.

The evidence for an association between dual diagnosis (major psychotic illness and drug or alcohol misuse) and violence is even stronger. Swanson et al (1990) found that if a research subject did not have schizophrenia and had not misused substances, then violence was reported in the preceeding 12 months in $2 \%$ of cases. If they had schizophrenia, but did not misuse substances, this rose to $8.3 \%$. If they had substance misuse alone then $21 \%$ reported being violent in the previous 12 months and if they had schizophrenia and misused substances then this increased to $30 \%$. A history of heavy use of alcohol was found in $48 \%$ of all subjects in the State Hospital, and $46.9 \%$ had used illegal drugs on at least one occasion, $9.5 \%$ by intravenous injection. In $16.2 \%$ of the cases alcohol and drug intoxication was implicated in the index offence.

\section{Symptoms}

Given the association between schizophrenia and violent offending in particular, researchers have looked to see whether specific symptoms were more likely to lead to aggression. In the high-security hospitals it was found that $54-75 \%$ of disturbed or offending behaviour leading to admission was psychotically motivated (Thomson et al, 1997; Taylor et al, 1998). Wessely et al (1993) in a study of 83 patients admitted to an acute psychiatric hospital with psychotic illness, mainly schizophrenia, found that $60 \%$ of participants reported at least one action based on a delusional belief and 20\% reported three or more. Delusions of a persecutory nature were most likely to be associated with aggression. Acting on delusions was associated with identification of evidence for it, especially in the past week; anxiety, sadness or fear arising from it; uncertainty if a belief was challenged; and actively seeking information to support or refute the delusional belief. Link \& Stueve (1994) have proposed that the presence of persecutory delusions and passivity phenomena, what they term 'threat control override' (TCO), make aggression a rational response to perceived threats. Patients with TCO were found to be twice as likely to have been violent during the previous 12 months than patients with other psychotic symptoms (Swanson et al, 1996). Other symptoms that have been related to aggression include delusional misidentification, erotomania and command hallucinations. 


\section{Box 1. Relevant publications}

Royal College of Psychiatrists (1996) Assessment and Clinical Management of Risk of Harm to Other People. Council Report CR53. London: Royal College of Psychiatrists.

Royal College of Psychiatrists (1998) Management of Imminent Violence. Occasional Paper OP41. London: Royal College of Psychiatrists.

Scottish Intercollegiate Guidelines Network (1998) Psychosocial Interventions and the Management of Schizophrenia. Edinburgh: Royal College of Physicians of Edinburgh.

\section{Assessment}

Patients with schizophrenia in a high-security setting require ongoing assessment of their illness, treatment resistance and risk. The assessment of risk examines violent episodes and considers any precipitating factors, including deterioration in mental state, life events, substance misuse, non-compliance and social instability (Royal College of Psychiatrists Special Working Party on Clinical Assessment and Management of Risk, 1996). Some have advocated an actuarial approach to risk assessment and tools have been developed such as the violence prediction scheme (Webster et al, 1994). This records information on historical factors such as alcohol misuse or school performance. Many clinicians have objected to this method as it is based on historical factors and fails to acknowledge any possible changes with age or treatment. Another powerful objection is the high false positive rate (i.e. those predicted to be at high risk of re-offending who did not) of $45 \%$ found by the authors of the tool. Webster et al (1997) have amalgamated historical, clinical and future risk factors in the HCR-20 risk prediction tool and this is the subject of current research.

Box 1 lists recent publications relevant to the assessment and management of patients with schizophrenia in a high-security setting.

\section{Management}

The patient's management should be centered on a multi-disciplinary team comprising psychiatric, nursing, social work, psychology and occupational therapy staff. Regular case conferences are held and treatment plans prepared. Treatment plans are aimed at: (a) improving mental state; (b) improving social functioning, self-care and self-esteem; (c) reducing aggressive or challenging behaviour; (d) promoting use of coping techniques; (e) encouraging community links; and (f) ongoing analysis of risk following each intervention (see Box 2).

\section{Legal issues}

Patients are detained under civil or criminal procedures. They are admitted from court, prison or other psychiatric hospitals. Their care incorporates the management of legal processes such as review of detention; opinions on psychiatric defences; recommendations to court regarding detention for assessment or final disposal; and preparation of reports for appeals against conviction, sentencing

Box 2. Management of schizophrenia in conditions of high security - summary

Multi-disciplinary approach

Assessment

Medication

Use of high-dose antipsychotic prescribing

Use of atypical antipsychotic drugs

Adjunctive treatment

Management of aggression

Environment and activities

Prevention

Control and restraint

Seclusion

Rapid tranquillisation

Psychosocial treatment

Structured day - work placements, daily living activities/skills, recreation

Family intervention

Education programmes

Cognitive-behavioural therapy

Treatment of substance misuse - combined dual diagnosis approach

Anger management

Anxiety management

Social skills training

Assertiveness training

Index offence work 
or detention. Evidence for the above may be required in written and/or oral format. Some patients have restrictions placed on their discharge on account of the nature of their offence, their previous history and likely risk of harm to the public as a result of their mental disorder, if released. Regular formal reports on restricted patients are required by the Home Office, Welsh Office or Scottish Office.

\section{Medication}

In 1993 over $60 \%$ of the State Hospital population was prescribed oral antipsychotic medication with an average daily chlorpromazine equivalent (ADCE) dose of $773 \mathrm{mg}$ (range 40-3600) and almost 50\% received a depot preparation (ADCE $972 \mathrm{mg}$, range 125-5000). Thirty seven per cent of patients were receiving combined oral and depot antipsychotic medication on a regular basis (ADCE $2033 \mathrm{mg}$, range 388-6300). In addition, 22 patients (9.4\%) were prescribed clozapine (mean $512 \mathrm{mg}$, range 250-900); $37(15.8 \%)$ an antidepressant; and 49 (20.9\%) an anticholinergic drug.

The Royal College of Psychiatrists' consensus statement on high-dose neuroleptic medication (Thompson, 1994) has had an effect on prescribing in the high-security hospitals. Polypharmacy and high-dose chlorpromazine equivalents were common. There was little evidence that either was beneficial. The number of patients receiving high-dose antipsychotic medication has fallen in one hospital from 35.1\% in May 1995 to 9.8\% in December 1998. Of the 21 patients who have stopped high-dose antipsychotic medication, 20 are receiving one atypical preparation and one a standard antipsychotic drug. Patients continuing to receive high-dose neuroleptic medication did so because of failure to respond to lower doses, aggression and lack of cooperation, and temporarily while medication was being adjusted (Barber et al, 1998). Trends in antipsychotic drug prescribing are shown in Tables 3 and 4 . With an increasing use of atypical antipsychotic medication, rather than depot preparations, it will be important to ensure that

Table 3. Atypical antipsychotic drug prescribing trends - State Hospital, Carstairs

\begin{tabular}{lrrrrr} 
Drug & \multicolumn{5}{c}{ Number of patients } \\
& 1995 & 1996 & 1997 & 1998 & 1999 \\
Clozapine & 25 & 33 & 33 & 35 & 45 \\
Risperidone & 23 & 22 & 16 & 22 & 25 \\
Olanzapine & - & - & 29 & 30 & 52 \\
Quetiapine & - & - & - & 7 & 10
\end{tabular}

Table 4. Traditional depot antipsychotic drug prescribing trends (M. Wright \& L. Beveridge, personal communication, 1999)

\begin{tabular}{lccccc} 
Drug & \multicolumn{5}{c}{ Number of patients } \\
& 1995 & 1996 & 1997 & 1998 & 1999 \\
Flupenthixol & 26 & 24 & 18 & 26 & 23 \\
Zuclopenthixol & 36 & 28 & 29 & 17 & 15 \\
Fluphenazine & 29 & 27 & 26 & 16 & 8
\end{tabular}

compliance is maintained as patients are transferred to other care settings.

The level of treatment resistance in high-security hospitals is the subject of current research. It is generally considered to be higher than the 5-20\% resistance level found in the total population with schizophrenia (Brenner et al, 1990), and may arise from non-compliance, incorrect diagnosis, intolerable side-effects, or excessive or inadequate dosage. Treatment refractoriness has been defined as ongoing psychotic symptoms with substantial functional disability and/or behavioural deviance which persists in well-diagnosed persons with schizophrenia despite reasonable pharmacological and psychosocial treatment that has been provided continuously for an adequate time (1-2 years).

The reasons given for choosing an atypical antipsychotic preparation in a high-security setting were refractory schizophrenia, intolerable side-effects, reduction in side-effects and marked negative symptoms. There is little evidence that these drugs improve the primary negative symptoms - anhedonia, alogia, flat affect and avolition - but their use may improve the secondary negative symptoms such as depression, sedation and extrapyramidal side-effects (King, 1998). Kane (1992) has shown that $30-60 \%$ of patients with treatment-refractory schizophrenia respond to clozapine, usually within six months. There is some evidence (Buckley et al, 1995; Rabinowitz et al, 1996) that clozapine may have an effect on aggression but Beck et al (1997) found no such effect for risperidone.

Secondary depression is common in this population and the use of antidepressants is beneficial. Carbamazepine is used as an adjunctive treatment in patients with aggressive or impulsive behaviour, or abnormal electroencephalogram. It induces the metabolism of some antipsychotic drugs and an increase in their dose may be required. Lithium is used less commonly as an adjunct in the treatment of aggression but is particularly useful in schizoaffective disorders. Benzodiazepines can provide sedation, enhance the effect of antipsychotic drugs and are useful in the treatment of neuroleptic-induced akathisia. However, the risk of addiction and side- 
effects must be considered. Electroconvulsive therapy is seldom used unless there is a strong affective component to an illness or a severe refractory state.

\section{Management of aggression}

Guidelines have been produced by the Royal College of Psychiatrists (1998) on the management of imminent violence.

A major aim of staff in high-security hospitals is to prevent violent episodes and to de-escalate those in progress. High-quality training is essential. Often, the use of voluntary time out in a quiet room or as-required medication avert aggression. A shortacting benzodiazepine, such as lorazepam, in combination with a rapid-acting neuroleptic drug, such as droperidol, is useful in acutely disturbed patients. At times, the use of control and restraint techniques may be required. Many wards in highsecurity hospitals have a specially designed seclusion room and policies for its use. Caution is required when a patient is shut in his or her own room and furniture and belongings removed; this is not 'voluntary time out' but seclusion. Mason (1998) studied all episodes of seclusion occurring over a one-year period in Ashworth Hospital $(n=823)$. Overall, $35 \%$ of patients were subject to seclusion, $30 \%$ of males and $68 \%$ of females. Females had on average 4.9 episodes of seclusion (average duration per episode 20 hours) and men 2.6 (average duration 3 days and 10 hours).

\section{Psychosocial treatment of schizophrenia}

High-security hospitals are institutions. Patients spend on average 4-7 years there. There is, therefore, considerable risk that institutionalised behaviour will develop, and this is known to have a deleterious effect on the prognosis of schizophrenia. Patients require access to activities, structure to their day and the opportunity to express themselves as individuals. In order to provide this activity, the highsecurity hospitals have occupational departments such as woodwork, arts and crafts, printing, stonework, gardens and laundry. Patients are given a choice as to which departments they would like to attend. In addition, there are education departments. Patients vary greatly in their abilities and some will require help with basic literacy skills, while others will pursue Open University courses. For most of us, life revolves around three basic activities: work, daily living tasks, and recreation. In the high-security hospitals patients are assessed on their ability to carry out basic living tasks, and recreational activities are available such as bowling or football. It is important to distinguish weekends and holidays from weekdays but at present this does not receive adequate attention. Activity is important, but it is also necessary not to overload patients or create an atmosphere on wards of high expressed emotion, as this is more likely to lead to relapse. Milieu therapy refers to a variety of psychosocial interventions in an in-patient setting. At a wardbased level it can involve simple interventions such as the creation and maintenance of a garden, video nights and takeaway meals.

National clinical guidelines have been published on psychosocial interventions in the management of schizophrenia (Scottish Intercollegiate Guidelines Network, 1998), which specify three major components: family/carer involvement; education programmes; and cognitive-behavioural therapy (CBT). The guidelines cite and systematically grade the evidence for each intervention in turn at different stages of a patient's illness.

The evidence suggests that family interventions involving support and education are effective but that intensive strategies are not necessary (Schooler et al, 1997). Family involvement can be difficult in the setting of a high-security hospital for geographical reasons or because an index admission event may have involved aggression towards a family member or created problems for the family in the community where they live. Social work departments play a crucial role in maintaining family links. Educational programmes, such as a coping with mental illness group, do exist but not uniformly nor always for families. A small study at Ashworth Hospital found a lack of support for and involvement of families by staff (McCann et al, 1996). It also highlighted the issue of publicity: families had often experienced adverse press coverage following the index event or, in some cases, following a visit home; alternatively, the negative coverage given to the high-security hospitals led to increased fears at the prospect of visiting.

Cognitive-behavioural therapy is a useful adjunct treatment for positive symptoms in patients with chronic schizophrenia (Tarrier et al, 1998). Target cognitions will vary and can include promoting insight, reducing distress, improving coping strategies and understanding, and specific symptoms and behaviour.

Psychoanalysis is considered by many to be inappropriate in the treatment of symptoms of schizophrenia. Hingley (1997) reviews the evidence for intensive psychodynamic therapy and those factors likely to lead to a good response. Poor responders included those with a history of substance misuse, violence, and poor work and 
social functioning, features common to many patients in high-security care.

\section{Additional therapies}

Many patients have an additional diagnosis as well as schizophrenia (see Table 1). Patient needs arising from these secondary diagnoses must be addressed. For example, it is acknowledged that, in the past, high-security hospitals have failed to adequately recognise the problem of substance misuse. This failure is currently being addressed and routine assessments and treatment programmes for dual diagnosis are being established. There will always be a fundamental problem, however, that patients cannot be tested fully in an essentially abstinent environment and that visits outwith the hospital are always in the presence of staff. An integrated approach in the treatment of psychosis and substance misuse is preferred that emphasises engagement and motivation and uses educational materials, skills training and relapse prevention techniques. Alcoholics Anonymous holds meetings within these hospitals.

Other treatment strategies include anxiety management and relaxation, assertiveness training, anger management and social skills training. Anger management programmes include education about aggression; self-monitoring of anger frequency, intensity and situational triggers; construction of a personal anger provocation hierarchy used for practicing coping skills; relaxation to reduce arousal and enable guided imagery training; cognitive restructuring by altering attentional focus, modifying appraisals and using self-instruction; training in behavioural coping, communication and assertiveness through role play; and practicing new anger-coping skills (Novaco et al, 2000).

Social skills training aims to provide patients with the skills to cope with their environment and there is evidence for its usefulness (Benton \& Schroeder, 1990). It generally takes place in groups using an educational approach, role play and modelling. Examples of specific skills to be addressed include communication and self-care.

It is important to address with patients the issues surrounding their index offence, where present. For example, the treatment of sexual offending requires in-depth assessment and acknowledgement of offence(s), and makes use of CBT principles. It can involve sexual education; control techniques such as recognition of high-risk times, people and places; understanding the cycle of abuse, which provides a structure for recognising the progression of feelings, thoughts and behaviour leading to a sexual assault; cognitive restructuring, which challenges and changes perceptions such as "the child wanted sex"; covert sensitisation, which aims to reduce deviant fantasies by pairing these with an aversive consequence and eventually replacing them with appropriate sexual imagery; victim empathy; and relapse prevention.

\section{Institutional issues}

The high-security hospitals by their very nature are closed institutions and all patients are detained. Admission to such a hospital with its distinctive security features and staff trained to manage disturbed behaviour can have a settling effect. It is important to recognise that people in such circumstances are vulnerable to the attitudes and actions of staff. Abuses have occurred in these settings and it is essential that all staff remain vigilant to this possibility. The existence of advocacy departments, rigorous complaints procedures and greater openness of the institutions themselves in terms of throughput of staff and visitors decrease the likelihood that such abuses will occur (see Box 3).

\section{Transfer procedures}

Patients in high-security hospitals are most commonly moved to psychiatric units of lower security but are also returned to prison or occasionally go directly into the community. The Care Programme Approach (Department of Health, 1990), Supervision Register (Department of Health, 1994) and Supervised Discharge/Community Care Order may all be used depending upon the location and legal status of the patient. One hospital has an expert panel to provide an independent opinion on the responsible medical officer's recommendation for transfer or continued hospital detention. For restricted patients transfer will require the consent of the relevant secretary of state, or first minister in Scotland. Patients may wait a long time for an appropriate placement elsewhere. It is essential that good communication is established between the

\section{Box 3. Institutional issues}

Institutionalisation

Patient vulnerability - vigilance for abuse Limited testing in a high-security setting Inappropriate placement in high security Lack of long-term medium secure beds 
transferring and receiving teams. Ideally a pretransfer case conference will take place and a treatment plan will be agreed. There is a need to address staff anxieties about some of these patients and the stigma that is attached to them. Trial leave may be used. Similar procedures should be used for those patients returning to prison.

\section{Follow-up}

There have been a number of studies examining criminal conviction following transfer from highsecurity psychiatric care. The most recent of these looked at 425 patients discharged from the special hospitals in 1982 and 1983 (Buchanan, 1998). After 10 years, $34 \%$ of patients had been convicted of an offence, $15 \%$ of a violent offence and $7.5 \%$ of a sex offence. These are lower levels of recidivism than exist in criminological populations. A younger age at discharge, legal detention for psychopathic disorder and previous convictions were all predictive of offending post-transfer.

\section{Conclusions}

Further research is required to examine the differences between those patients with schizophrenia who manifest dangerous, violent or criminal propensities and those who do not. Using these factors it may be possible to construct an early intervention programme for those deemed to be at greater risk. Risk, however, can be reduced and managed but never eliminated.

From a service management perspective it would be preferable if patients in high-security care resided in smaller residential units. It is important to develop medium secure care in Scotland and Northern Ireland, and to extend the existing provision in England and Wales to include long-term medium secure beds.

\section{References}

Barber, J. M., Connaughton, J. \& Wright, M. (1998) Monitoring patients on high-dose antipsychotics. Psychiatric Bulletin, 22, 671-674

Beck, N. C., Greenfield, S. R., Gotham, H., et al (1997) Risperidone in the management of violent, treatmentresistant schizophrenics hospitalized in a maximum security forensic facility. Journal of the American Academy of Psychiatry and Law, 25, 461-468.

Benton, M. K. \& Schroeder, H. E. (1990) Social skills training with schizophrenics: a meta-analytic evaluation. Journal of Consulting and Clinical Psychology, 58, 741-747.
Brenner, H. D., Dencker, S. J., Goldstein, M., et al (1990) Defining schizophrenia refractoriness in schizophrenia. Schizophrenia Bulletin, 16, 551-561.

Buchanan, A. (1998) Criminal conviction after discharge from special (high security) hospital. Incidence in the first 10 years. British Journal of Psychiatry, 172, 472-476.

Buckley, P., Bartell, J., Donenwirth, K., et al (1995) Violence and schizophrenia: clozapine as a specific antiaggressive agent, Bulletin of the American Academy of Psychiatry and Law, 23, 607-611.

Department of Health (1990) 'Caring for People': The Care Programme for People with Mental Illness Referred to Specialist Psychiatric Services. Huywood: Department of Health.

Department of Health (1994) Introduction of Supervision Registers for Mentally Ill People. Circular SHG (94) 5. London: Department of Health.

Eronen, M., Tiihonen, J. \& Hakola, P. (1996) Schizophrenia and homicidal behaviour. Schizophrenia Bulletin, 22, 83-89.

Hingley, S. M. (1997) Psychodynamic perspectives on psychosis and psychotherapy. II: Practice. British Journal of Medical Psychology, 70, 313-324.

Hodgins, S., Mednick, S. A., Brennan, P. A., et al (1996) Mental disorder and crime: evidence from a Danish birth cohort. Archives of General Psychiatry, 53, 489-496.

Humphreys, M. S., Johnstone, E. C., MacMillan, J. F., et al (1992) Dangerous behaviour preceding first admissions for schizophrenia. British Journal of Psychiatry, 161, 501505.

Kane, J. M. (1992) Clinical efficacy of clozapine in treatmentrefractory schizophrenia: an overview. British Journal of Psychiatry, 160 (suppl. 17), 41-45.

King, D. J. (1998) Atypical antipsychotics and the negative symptoms of schizophrenia. Advances in Psychiatric Treatment, 4, 53-61.

Link, B. G. \& Stueve, A. (1994) Psychotic symptoms and the violent/illegal behaviour of mental patients compared to community controls. In Violence and Mental Disorder: Developments in Risk Assessment (eds J. Monahan \& H. J. Steadman), pp. 137-159. Chicago, IL: University of Chicago Press.

— \& - (1998) New evidence on the violence risk posed by people with mental illness: on the importance of specifying the timing and the targets of violence. Archives of General Psychiatry, 55, 403-404.

Maden, A., Curle, C., Meux, C., et al (1993) The treatment and security needs of patients in special hospitals. Criminal Behaviour and Mental Health, 3, 290-306.

Mason, T. (1998) Gender differences in the use of seclusion. Medical Science and Law, 38, 1-9.

McCann, G., McKeown, M. \& Porter, I. (1996) Understanding the needs of relatives of patients within a special hospital for mentally disordered offenders: a basis for improved services. Journal of Avdanced Nursing, 23, 346-352.

Miller, P. McC., Johnstone, E. C., Lang, F. L., et al (2000) Differences between patients with schizophrenia within and without a high security psychiatric hospital. Acta Psychiatrica Scandinavica, in press.

Modestin, J. \& Ammann, R. (1996) Mental disorder and criminality: male schizophrenia. Schizophrenia Bulletin, 22, 69-82.

National Health Service Executive (1999) The Safety and Security in Ashworth, Broadmoor and Rampton Hospitals Directions. HSC 1999/150. Leeds: NHSE.

Novaco, R., Ramm, M. \& Black, L. (2000) Anger treatment and offenders. In Handbook of Offender Assessment and Treatment (ed. C. Hollin). Chichester: John Wiley \& Sons, in press.

Rabinowitz, J., Avnon, M. \& Rosenberg, V. (1996) Effect of clozapine on physical and verbal aggression. Schizophrenia Research, 22, 249-255.

Royal College of Psychiatrists Special Working Party on Clinical Assessment and Management of Risk (1996) Assessment and Clinical Management of Risk of Harm to Other People. Council Report CR53. London: Royal College of Psychiatrists.

- (1998) Management of Imminent Violence. Clinical Practice Guidelines to Support Mental Health Services. Occasional Paper OP41. London: Royal College of Psychiatrists. 
Schooler, N. R., Keith, S., Severe, J., et al (1997) Relapse and rehospitalisation during maintenance treatment of schizophrenia: the effects of dose reduction and family treatment. Archives of General Psychiatry, 54, 453-463.

Scottish Intercollegiate Guidelines Network (1998) Psychosocial Interventions in the Management of Schizophrenia. A National Clinical Guideline. SIGN Publication Number 30. Edinburgh: Royal College of Physicians of Edinburgh.

Steadman, H. J., Mulvey, E. P., Monahan, J., et al (1998) Violence by people discharged from acute psychiatric inpatient facilities and by others in the same neighborhoods. Archives of General Psychiatry, 55, 393-401.

Swanson, J. W., Holzer, C. E., Ganju, V. K., et al (1990) Violence and psychiatric disorder in the community: evidence from the Epidemiologic Catchment Area surveys. Hospital and Community Psychiatry, 41, 761-770.

—, Borum, R. \& Swartz, M. S. (1996) Psychotic symptoms and disorders and the risk of violent behaviour in the community. Criminal Behaviour and Mental Health, 6, 309-329.

Tarrier, N., Yusupoff, L., Kinney, C., et al (1998) Randomised controlled trial of intensive cognitive behaviour therapy for patients with chronic schizophrenia. British Medical Journal, 317, 303-307.

Taylor, P. J., Leese, M., Williams, D., et al (1998) Mental disorder and violence. A special (high security) hospital study. British Journal of Psychiatry, 172, 218-226.

Thompson, C. (1994) The use of high-dose antipsychotic medication. British Journal of Psychiatry, 164, 448-458.

Thomson, L. D. G., Bogue, J., Humphreys, M., et al (1997) The State Hospital Survey: a description of psychiatric patients in conditions of special security in Scotland. Journal of Forensic Psychiatry, 8, 263-284.

Tiihonen, J., Isohanni, M., Rasanen, P., et al (1997) Specific major mental disorders and criminality: twenty six year prospective study of the 1966 northern Finland birth cohort. American Journal of Psychiatry, 154, 840-845.

Webster, C. D., Harris, G., Rice, M., et al (1994) The Violence Prediction Scheme, Assessing Dangerousness in High Risk Men. Toronto: Centre of Criminology, University of Toronto.

_ Douglas, K., Eaves, D., et al (1997) HCR-20: Assessing the Risk of Violence, Version 2. Burnaby, BC, Canada: Mental Health, Law and Policy Institute, Simon Fraser University, in cooperation with the British Columbia Forensic Psychiatric Services Commission.

Wessely, S., Buchanan, A., Reed, A., et al (1993) Acting on delusions. I: Prevalence. British Journal of Psychiatry, 163 69-76.

- Castle, D., Douglas A. J., et al (1994) The criminal careers of incident cases of schizophrenia. Psychological Medicine 24, 483-502.

\section{Multiple choice questions}

1. High-security psychiatric care in the UK:

$\mathrm{a}$ is defined by requirement for length of stay of greater than 2 years

$\mathrm{b}$ is jointly administered by the NHS and prison service

c contains up to $50 \%$ of patients who do not have to be there

d contains patients who are all formally detained

e contains a majority of patients with a diagnosis of schizophrenia and comorbid personality disorder.

2. Violence in patients with mental disorder:

a is associated with schizophrenia

b is associated with schizophrenia and comorbid substance misuse

c is always psychotically motivated

$\mathrm{d}$ is predictable using an actuarial approach

e occurs in one-fifth of cases prior to a first admission for schizophrenia.

3. Neuroleptic medication for the management of schizophrenia in high security:

a is used less commonly in preference to mechanical restraint

$\mathrm{b}$ is routinely given as a depot preparation to ensure compliance

c is used in addition to electroconvulsive therapy for the treatment of psychosis and aggression

d makes use of clozapine, which has an antiaggressive effect

e makes use of risperidone, which has an antiaggressive effect.

4. Useful psychosocial treatments of schizophrenia in high security include:

a cognitive-behavioural therapy

b psychoanalysis

c intensive family therapy

$\mathrm{d}$ anger management

e social skills training.

5. Patients with schizophrenia in a high-security setting:

a are more likely to have physical health problems

$\mathrm{b}$ are secluded on a voluntary basis

c were psychotic at the time of the index event leading to admission in the majority of cases

$\mathrm{d}$ face delays in transfer due to lack of suitable psychiatric facilities

e have a $30 \%$ post-transfer violent re-offending rate.

MCQ answers

\begin{tabular}{|c|c|c|c|c|}
\hline 1 & 2 & 3 & 4 & 5 \\
\hline F & a $T$ & a $F$ & a $T$ & a 7 \\
\hline F & b $T$ & b F & b $F$ & b $F$ \\
\hline$T$ & c $F$ & c $F$ & c $F$ & c 1 \\
\hline$T$ & d F & d $T$ & d $T$ & d \\
\hline F & e $T$ & e $F$ & e $T$ & e \\
\hline
\end{tabular}

